\title{
Prospective analysis of the impact of pandemic in Industry 4.0
}

\author{
Georgios Andreadis ${ }^{1}$ and Ana Isabel Quirós Gámez, ${ }^{2 *}$ \\ ${ }^{1}$ Laboratory for Machine Tools and Manufacturing Engineering, Mechanical Engineering \\ Department, Aristoteles University of Thessaloniki, Greece \\ ${ }^{2}$ Escuela Técnica Superior de Ingeniería. University of Seville, Spain
}

\begin{abstract}
The world population in 2020 was estimated at 6.070 million and is projected to grow to around 9 billion by 2050 . The evolution and transformation of society in the technological field is a challenge due to its rapid evolution and the social consequences it triggers. The pursued aim is the prospective analysis of diverse scenarios directed by Industry 4.0 macro-drivers, breeding cornerstones for the purpose to presage future pandemic backdrop. In accordance with the evolution of the force for change due to the analyzed factors, a series of recommendations and future work is elaborated to create a weapon to confront this possible context.
\end{abstract}

\section{Introduction}

The analysis of miscellaneous probable scenarios predictions impeached to the evolution of the industry or the setback of the latter is the focus of this research. Taken hand in hand with the social evolution, these progressions will entail in the adaptation of Industry 4.0 or even the evolution towards a fifth industrial revolution linked to building a bridge to the Japanese concept of society 5.0. It ought to be emphasized that the proposed scenarios are predictions based on a prospective analysis, not measurements.

The structure of the document consists of an initial section explaining the methodology for the classification, where engines of changes related to Industry 4.0 will be examined. Likewise, influential actors will be identified in this epigraph. Consecutively, the substantial point of the analysis is found in the codification of different situations governed by the development of the aforementioned mega drivers. To conclude, recommendations in a pandemic prospect would divert the circumstance with the subsequent improvement are assessed

\section{Methodology}

One of the uncertainties sought for noteworthy prediction is what is to come. Topicality some authors and theories attest that the future can be built and not merely be predicted. Under this prism, there are two types of futures within the discipline: presumable future and potential future [1].

\footnotetext{
*Corresponding author: anaquirosgamez@hotmail.com
} 
Everything is happening at the present has an explanation in the past. Along these lines, predictive analysis can be accomplished in the event of a pandemic based on COVID-19 and consider its repercussion in possible scenarios. Thus, leaving evidence of the importance of good decision making.

\subsection{Driver identification}

The purpose of this section is to identify the macro drivers and micro drivers considered as change motors that are skilled to prompt the different scenarios. These megatrends variables are systematized and schematized in Table 1, which will be explained in the subsequent subsections.

Table 1. Analytical resources

\begin{tabular}{|c|c|}
\hline Macro drivers & Micro drivers \\
\hline \multirow{3}{*}{ Risks } & Technological risks \\
\hline & Natural disaster \\
\hline & Human risks \\
\hline \multirow{3}{*}{ IoT } & Low consumption \\
\hline & Device and connexion compatibility \\
\hline & Network traffic problems \\
\hline \multirow{3}{*}{ Artificial Intelligence } & Biosensors \\
\hline & Encryption and Data Integration \\
\hline & Machine learning \\
\hline \multirow{2}{*}{ Big Data } & Quality of the data \\
\hline & Secure storage \\
\hline \multirow{2}{*}{$\begin{array}{c}\text { International Political } \\
\text { Agenda }\end{array}$} & R\&D \\
\hline & Centralization and Inter-community agreements for innovation \\
\hline \multirow{3}{*}{ Cloud Computing } & Remote medical assistance and telework development \\
\hline & Social Networks \\
\hline & Online commerce \\
\hline \multirow{2}{*}{ Society 5.0} & Adaptation of new technologies and technological education \\
\hline & Access to privacy \\
\hline
\end{tabular}

\subsubsection{Risks}

Risks are substantial for impact analysis. The two great threats confronting the course of humanity are incorporated, likewise including a third pivotal factor:

-Nature, where it is enclosed both natural, climatic, and biological catastrophes. It is a decisive engine of change for any conceivable future.

-The human itself. It is considered the risk of social crisis as financial. Extraordinary improvement of the industry could infer the vanishing of manual work and the nonadjustment of society. Nuclear disasters or warfare are also enveloped, which would be a strong blow and would undoubtedly be determining variables respect any prediction.

-The technological risk, both the hardware and software ones, it is contemplated cybersecurity, cloud computing, or infrastructure servers' risks.

\subsubsection{Internet of Things}

The improvement of the Internet of Things is also a megatrend. The possibility of gathering data through sensors on mobiles and the interconnection of devices is a fundamental piece to fit in the development of probable futures. In the case of pandemics, it could provide 
valuable data such as information on confinement and population movement. Tracing the origin of the disease, the spread and the contagion of the virus through patient zero are factors that could be controlled because of the development of this engine of progress.

\subsubsection{Artificial Intelligence}

Progress in this field is an underlying element. The development of smart algorithms for useful samples collection to use in future pandemics or the advance in machine learning are imprescriptible components of metamorphosis. The capacity to encrypt and protect this information is one of the security challenges against cyberattacks. Notwithstanding within artificial intelligence, it could be listed an enormous volume of drivers for change, but centering in a pandemic scenario, it will be distinguished the development of biosensors, detectors, and forecasters of the disease to contain the spread of the virus. The propulsion of $3 \mathrm{D}$ printing in addition to scanning technologies with the noteworthy capacity to cover demand will also compound a weapon to combat dearth. The virtual reality will allow the flow of teleworking and the development of cloud computing macrotrends.

\subsubsection{Big Data}

The enormous volume of information that is dealt with today assumes a crucial role in society. Useful data as referenced above is critical to forestall and has the power to predict future occasions. The real-time integration of the number of infected people from the official sources, both medical and security, is essential for good dynamic decision-making against the battle with the virus.

\subsubsection{International Political Agenda}

Government entities have an essential role in managing the ailment. GDP is a hazard factor that can cause a global collapse of the bourse and financial exchange; therefore, a monetary emergency would emerge. The results of a crisis can trigger the stoppage of the influencer drivers considered. Institutions need to oversee food and personal protective equipment deficiencies, as well as the possible appearance of mafias. Investment in R\&D for the development of Industry 4.0 will be unequivocal in the evolution towards a modern revolution and the intra-community agreement towards progress will make the difference between scenarios.

\subsubsection{Cloud Computing}

Amidst isolation, life goes on, albeit in a digitally and not personal way. Owing to this macro driver, the new normality can be lived ceaselessly in time without venturing out from home. The connectivity between people through social networks, the development of the telecommuting, or computerized business will make it conceivable to maintain a strategic distance from monetary insolvency and market stoppage. Moreover, because of the advancement of remote clinical care, a large mass of undiagnosed patients is avoided in hospitals and can be followed up on those already diagnosed in a non-dangerous phase of the disease. This is a fundamental service. 


\subsubsection{Society 5.0}

Finally, it is mentioned one of the most determining factors in those different situations: the evolution of the population. This Japanese concept of Society 5.0 is referenced owing to the development of the pandemic has accelerated various patterns and could trend a bridge to this new intelligent lifeway. The objective pursued is to situate individuals at the focal point of all ongoing technological transformation, taking the German concept of Industry 4.0 to a higher level and offering a path to a coordinated and interconnected society. As per this vision, the sophisticated integration of cyberspace (data) and physical space (genuine world) will be a reality and will shape human-centered socio-economic and technological models. One of the keys to ensuring success in its execution is the change in mindset and education in innovations. Thusly, we would move from Information Society 4.0 to coordinated, integrated, and sustainable sophistication [2].

\subsection{Actors identification}

In this section, the actors that are part of the evolution and financing within the Industry 4.0 paradigm are distinguished. In this concise portrayal of them, it is considered the European Union as the focal point of the investigation.

\subsubsection{Internal Actors}

It is differentiated between economic operators, such as influential investment and development companies, and politician agents made up of every nation's governments and the European Union as their coordinator. The administration of research and development (R\&D) is an overriding factor to emphasize, whose burden is on the internal actor's management. Inside this gathering, it is additionally incorporated specialists' confederations, researchers, and engineering designing advancement entities.

\subsubsection{External Actors}

The actors who influence the transformation of the object of study but have no direct impact on the development of Industry 4.0 in Europe are englobed in this paragraph. It is incorporated supranational entities, such as the UN, specifically UNIDO (United Nations Industrial Development Organization). Emerging markets, where it is included China, Japan, and Russia (which is also a border with Europe). It is also considered the US, whose Industry 4.0 market between 2016-2023 will develop at a CAGR of 12.9\%. PR Newswire said, "The United States, the world's second-largest manufacturer, with a 2017 industrial output reaching a record level of $\$ 2.2$ trillion, will apply Industry 4.0 technologies to replace China as the world's largest manufacturer." [3].

\section{Analysis of potential future scenarios}

Three possible future situations depending on the combination of furtherance of the recognized drivers are introduced. The + and - symbols imply the level of positivity and negativity of the drivers.

Table 2. Codification of drivers.

\begin{tabular}{|l|c|c|c|c|c|c|c|}
\hline & Risks & IoT & AI & Big Data & IPA & $\begin{array}{c}\text { Cloud } \\
\text { Computing }\end{array}$ & $\begin{array}{c}\text { Society } \\
5.0\end{array}$ \\
\hline
\end{tabular}




\begin{tabular}{|c|c|c|c|c|c|c|c|}
\hline Severe Scenario & + & - & - & - & - & + & - \\
\hline Status Quo & - & + & - & - & + & + & - \\
\hline Positive Scenario & - & + & + & + & + & + & + \\
\hline
\end{tabular}

\subsection{Severe Scenario}

The uncontrolled pandemic situation, where the attack rate is $80 \%$ [4]. The lasting decline in the labor flow is around $0.8 \%$ and along these lines, the demand for labor decreases considerably. A case like the Spanish flu of 1918-1919 is confronted. Containing the disease becomes impossible and sanitary breakdown occurs (health personnel contagion due to lack of PPE and the uncontrolled number of patients in an advanced stage of the disease). Deficiencies and inadequacy of manufacturing are reached due to the majority of non-automatization of the business and the insufficiency of adaptation. Inconceivability advancement of online work because of the requirement for an administrator to operate a machine occurs. The economy and the stock market plummet due to confinement and governments do not have the capacity to finance technological development. The GDP of nations falls significantly. In a vulnerable circumstance, the risk from cyberattacks enhances.

Solidarity movement is generated with $3 \mathrm{D}$ sanitary material printing however it is insufficient performance due to the poor development of this technology. The data (the contagions detected) are stored in the cloud and they tend to be considered for preventing a future pandemic, despite they are not genuine in order of magnitude. Communication among individuals and nations would likewise be secured covered because of correspondence communication networks and mobile telephony networks. Industry 4.0 standard covers a super communicated but not integrated and intelligent society. The technology exists yet is not executed or financed.

\subsection{Status Quo}

McKibbin \& Fernando consider attack rates (share of the population who become sick) in the range $1-30 \%$ and case-fatality rates (share of those infected who die) in the range $2-3 \%$ [5]. Industry 4.0 technologies have the capability of providing better-computerized solutions for our daily lives during this crisis. Online commerce and the voluptuous industry fulfill demand. There is no lack due to the transformation of operability in smart factories (environments where are used to make perfumes are transformed to manufacture hydroalcoholic gel during COVID-19 pandemic period). The possibility of teleworking is limited, and remote medical assistance or control of population movements are a reality thanks to the IoT. Exists a pure state of the Industry 4.0 concept neutral (neither in advance nor in reverse bias). It could be argued in this circumstance an acceleration of the process of adoption of Industry 4.0 to a situation of abnormality, passing to a more mature state of the German concept.

\subsection{Positive Scenario}

Verity et al. evaluated for this situation a disease casualty proportion of $0.145 \%(0.08-0.32)$ for individuals younger than 60, and 3.28\% (1.62-6.18) for individuals matured at least 60 [4]. Pandemic regulation is practiced rapidly because of valuable data store in the cloud from past pandemics and legitimate preventive and control of this infection. The breakdown is avoided owing to the investment funds for Industry 4.0 and the development of new technologies, which is the first step towards communication integration and distribution. 
Society would thus be able to get closer to Industry 5.0 and therefore it would be immersed in awareness mentality getting closer to the super-intelligent population, almost involved in the Japanese concept of society 5.0.

\section{Conclusion}

Going forward, the medical industry would grow and the requirement of digitization of certain sectors is indispensable. An intelligent, centralized, and digitized healthcare system mitigate the risks of collapse and guarantees coverage of remote populace areas. Emphasis is placed on the development of smart manufacturing in order to assemble, conveyance, archive, and analyze data framework. Advances in Industry 4.0 will provide infrastructures to control the proper isolation of the patients, accelerating the medication fabricating and treatment process control. Along with, "formulation of national strategies and integration of the government's promotion system". Counting the architecture of a 'practical IoT system' and a think-tank function [2]. Regulated and centralized administrative digitization and the breaking of the digital cleavage are essential challenges to face the situation.

The consequences will rely upon the implication of the different elements and circumstances. The prerogative society is submerged in is a pandemic scenario, so consequently, recommendations so as to fortify the entrepreneurial framework in case of a resurgence of similar situations, are designed. Industry 4.0 is positioned at the epicenter of the study to improve production capacity and trace a channel to future developments. Given this circumstance, it is anticipated a noticeable requirement to fortify the execution of the existent technology (it has been evidenced that the impact of the crisis on the pandemic has been substantially less aggressive in automated companies due to their ability to production adaptation). The need to reduce the risk factors mentioned above as far as possible so that to provide stability for a favorable sustainable environment is pivotal. Hence, persuading the cooperation of the public and private sectors is indispensable in a progressively and successful environment. To end up, and parallel that, reinforce intra-community network financing projects is a vigorous motor of changes in order to further the potential of the individual-technology relationship in fostering the enhancement of the quality of life of all people through a super-smart society [6].

\section{References}

1. Espejo, V. Megatendencias: un análisis del estado global (Análisis de tendencias del mundo). C.N. P. E (2016).

2. Keidanren. Society 5.0: The big societal transformation plan of Jap.

3. HSRC. The U.S. and China Invest Heavily in Industry 4.0 Technologies to be the World's Largest Manufacturer.

4. Verity, R., Okell, L. C., Dorigatti, I., Winskill, P., Whittaker, C., Imai, N., CuomoDannenburg, G., Thompson, H., Walker, P. G. T., Fu, H., Dighe, A., Griffin, J. T., Baguelin, M., Bhatia, S., Boonyasiri, A., Cori, A., Cucunubá, Z., FitzJohn, R., Gaythorpe, K., ... Ferguson, N. M. Estimates of the severity of coronavirus disease 2019: A model-based analysis. The Lancet Infectious Diseases. (2020)

5. Del Rio-Chanona, R. M., Mealy, P., Pichler, A., Lafond, F., \& Farmer, D. Supply and demand shocks in the COVID-19 pandemic: An industry and occupation perspective. (2020).

6. Serpa, S., \& Ferreira, C. Society 5.0 and Social Development [Preprint]. (2018) 